\title{
Mobile Devices: Opportunities for Users with Special Needs*
}

\author{
Enrico Bertini and Stephen Kimani \\ Università di Roma "La Sapienza", Dipartimento di Informatica e Sistemistica \\ Via Salaria 113, 00198 Roma, Italy \\ \{bertini,kimani\}@dis. uniroma1.it
}

\begin{abstract}
Breakthroughs in mobile and wireless technologies have revolutionized the world in virtually every aspect. While much work has been and is being done regarding the opportunities and challenges arising from these technologies, much less exists on the unique opportunities and implications the same devices present and raise to users with special needs. Furthermore, the existing little work is normally specific to only a certain type of disability or device. While addressing a specific type of disability or mobile device has its place, it is also significant to ensure that one is operating based on a holistic perspective/framework of the entire audience of the disabled mobile device users.
\end{abstract}

\section{Introduction}

Advances in mobile and wireless technologies have, to a great extent, revolutionized the world. A user carrying out one or many parallel activities, from virtually anywhere at anytime while at the same time interacting with other user(s) has become commonplace. While the opportunities, challenges and benefits stemming from mobile device usage are clearly evident and widely published, much less exists about the unique opportunities and requirements mobile devices present and pose to users who have special needs. There have been and are various efforts pertaining to mobile devices and users with special needs. Most of the efforts have addressed the two arenas as two independent or separate aspects. Surprisingly enough, the few research efforts that cover both arenas are normally specific to only a certain type of disability (or even device). We should quickly point out that we do commend such efforts as they venture to ensure that a certain type of disability (or device) is addressed to reasonable depth. While we acknowledge that addressing only a particular type of disability (or mobile device) has its place, we do however find it also worth to operate based on a rather comprehensive or holistic framework of the entire audience of the disabled mobile device users. A few related efforts are discussed in the sequel.

\footnotetext{
* The work presented in this paper is funded by the Italian Ministry for Education, University and Research (MIUR) through the MAIS (Multichannel Adaptive Information Systems) project. More information can be found at http://black.elet.polimi.it/mais.
} 
Fellbaum's enlightening work on general speech and hearing technology [1] has some hints on the same technology with respect to the deaf and the speech impaired. Although Freitas et al [2] focus on mobile phones, their work does partially propose various ideas that can be helpful toward meeting the special needs of the disabled and the elderly. Abascal et al [3] explore various implications on the elderly, emanating from the use of mobile phones. The research effort [4] presents display techniques to aid visually impaired users by the enhancement of the screen objects whereas [5] focuses on the use of haptic interfaces for motionimpaired users. Though existing accessibility standards e.g. the ones proposed by ETSI ${ }^{1}$ often pertain to the wide arena of Information and Communications Technologies (ICTs), they have resourceful information that can be exploited in mobile computing to support users with special needs.

The primary purpose of this paper is to describe the unique opportunities that mobile devices present to users with special needs such as the disabled/impaired, the elderly, and the sick. Rather than focusing on a certain type of disability or mobile device, the paper presents the discussion based on a more holistic perspective of this type of user audience.

\section{Opportunities for Users with Special Needs}

This section starts by presenting an analysis of the unique opportunities for users with special needs that are offered by mobile devices. It should be pointed out that such users do have special requirements. Toward ensuring that their special needs are catered for, this section later describes various approaches that can be used to address the special challenges facing such users in the context of mobile computing.

\subsection{What the Mobile Devices Offer to the Disabled}

The core benefit concerning the use of mobile devices for disabled users is that the devices' mobility and connectivity can assure the users a constant "companion". The applications of mobile devices for disabled users fall in the following three classes:

Mobiles as an Aid to Carry Out Functions: Mobile devices can host applications that permit the users to be actively involved in everyday activities, as though they were not disabled e.g. the physically impaired can use the devices to remotely instruct PCs [6] or even lifts, doors, ATMs, etc.

Mobiles as a Means to Communicate: Since the devices are mobile and can be connected, the disabled users can always carry them and communicate with them. Communication from virtually anywhere is hence rendered easier. In fact, the devices can function even when the user's inherent communication functionalities are impaired [7].

$\overline{{ }^{1} \text { http://www.etsi.org }}$ 
Mobiles as Assistants: Mobile devices can exploit the context which is in itself a great opportunity for the disabled e.g. advising the users of particular dangers, acting as guides, etc.

Besides the foregoing direct opportunities deriving from the increase in availability, there are also some indirect opportunities arising from the use of mobile devices including:

Non-conventional Interactions: Many of the standard settings are no longer applicable in mobile computing. The foregoing challenge is indeed an opportunity to carry out corresponding studies. For instance, investigating relevant interaction paradigms that take into account users with special needs. (Section 2.2 has more information on such aspects.)

Democratization: Through mobile devices, computing technology is no longer a territory of a selected few. In part, the extended user audience stems from the declining cost of mobile and wireless technologies, and the type of operations/work mobile devices are designed to support. Consequently, more disabled users have managed to access or acquire the devices. (Section 2.2 has more information on the economic aspect.)

\subsection{Toward Fulfilling the Users' Special Needs}

As it was observed in Section 2.1, mobile devices present many opportunities for the elderly, the impaired/disabled, and the sick. Nonetheless, the users have special needs/requirements. In the sequel, is a discussion of various approaches that can be adopted toward fulfilling the special needs of these users while interacting with mobile devices.

Alerting Techniques: Mobile devices should use alerting techniques that can be comprehended by users with special needs. Alerts are often signaled in an audible (sound), visible (flashing light) and felt (vibrating transducer) form. The alert is also coupled with some message/cue (e.g. audio, graphical/icon, text) pointing out what the alert is for.

Wearability: The market of mobile devices and wireless technologies offers a lot toward rendering mobile devices wearable. With wearability, the disabled user need not worry about physically reaching or misplacing the device. An interesting example is the wearable PDA for the blind ${ }^{2}$.

\footnotetext{
${ }^{2}$ http:// www. freedomscientific.com
} 
Non-conventional Input and Output Paradigms Auditory Services and Interfaces: Despite the challenges that come with auditory interfaces [1], they still can be very useful to the disabled especially the physically impaired and/or the visually impaired. It is important to ensure that the voice interface has a volume control that is easily accessible. Speech input and output devices (hearing aids) for mobile devices do exist and some can actually be worn. There are also various services that go together with voice interfaces. For any audio output/input, it is highly desirable, though non-trivial, that the system be able to make available the corresponding textual output/input and vice versa, if the situation demands so. On the whole, it might be worth exploring the relevance of enabling mobile devices to support/handle input and output in various data formats and being able to interpret from any of the formats and convert to any of the formats.

Visual Interfaces: The small screen size of mobile devices has been a major challenge in ubiquitous computing. It would be interesting to see how existing proposals could be useful in addressing problems that the disabled experience when using the small screens. It might be worth investigating the effectiveness of developing customized/personalized visual output devices for users with special needs. Using pictorial representations to represent functionalities offered by the mobile device can be helpful especially to the cognitively impaired. On another note, the mobile device could offer an optional and special character set with fewer characters and that of a larger size than the standard set.

Keyboards/keypads: As far as the keyboard/keypad is concerned, special keyboards/keypads may be considered. The keyboards/keypads could still offer the traditional 5-key dot/cue. It could be important to providing tactile, audio, and visual cues on the keyboard/keypad. Moreover a strong contrast of the keys and the labels should be adopted; in fact contrasting should be effectively applied to the whole device. Further, disabled users might find a simple keyboard/keypad having only a few (and slightly bigger) key buttons reasonably sufficient. Such a keyboard/keypad would enable the users (including the cognitively impaired) to easily/quickly perform operations.

Simple and Shallow Menu/Command System: The mobile device should enable the user to access certain functionality without having to go through a lot of steps. The menu/command system should be easy to understand, simple and direct, with help (described in the next discussion on Multilevel assistance) whenever necessary.

Multilevel Assistance: This assistance/help facility may comprise two layers for instance local/device-based help and remote/service center-based help. On the whole, help should be directly/easily accessible, interactive and easy to understand/interpret. Some circumstances would necessitate the automatic activation of help (e.g. connectivity status, battery status). Consider in some detail the latter example of battery status. If the device's battery needs recharging, the device could use the aforementioned alerting techniques. If the user does not 
respond to the alert (local help), the device could activate service center-based help by notifying the center about the situation.

User Interface Considerations: The user interface could be designed in a manner such that it adapts its interaction mechanism(s) and presentation style(s) in order to meet the specific needs of a particular disabled user. Such a feature essentially requires that various aspects of the particular user be modeled. Such aspects include: the user, the information, and the context. Moreover, it is worth embracing the design for all or universal design approach [8, 9].

Economic Aspects: It is gratifying to observe that the prices of mobile devices and wireless technologies are decreasing. However, the prices are still fairly high for the disabled users who normally are financially disadvantaged. Various stakeholders regarding matters pertaining to pricing and social welfare e.g. government(s), organizations, etc should help to make the mobile devices affordable to users with special needs (e.g. subsidized prices, donations). It is interesting to realize that, though design for all may be costly in the short-term, it is ultimately beneficial not only to the users but also to businesses.

\section{Conclusions}

In this paper we have addressed the question of the unique opportunities mobile devices offer to the disabled. We have also presented various approaches that can be used toward meeting the special needs of the disabled. A lot of work is yet to be done and the success of the next generation of user interfaces and devices depends in part on our understanding of how disabled users can benefit from these new technologies and in part on the evolution of markets and political/sociological directions. There is still much to be done, novel and otherwise, toward supporting the exploitation of this great opportunity and addressing respective challenges.

\section{References}

[1] K. Fellbaum: Speech and Hearing Technology: A Critical Review. In: Proceedings of COST 219 Seminar on Speech and Hearing Technology. (2000) 487, 489

[2] D. Freitas and J. Gjöderum and G. Hellström and M. Soede: Mobile Telephony. In: Telecommunications for All. (1995) 487

[3] J. Abascal and A. Civit: Mobile Communication for Older People: New Opportunities for Autonomous Life. In: Proceedings of EC/NSF Workshop on Universal Accessibility of Ubiquitous Computing: Providing for the Elderly. (2001) 487

[4] Jacko, J.A., Scott, I.U., Sainfort, F., Barnard, L., Edwards, P.J., Emery, V.K., Kongnakorn, T., Moloney, K.P., Zorich, B.S.: Older adults and visual impairment: What do exposure times and accuracy tell us about performance gains associated with multimodal feedback? In: Proceedings of Conference on Human Factors in Computing Systems, ACM Press (2003) 487 
[5] Hwang, F., Keates, S., Langdon, P., Clarkson, P.J.: Multiple haptic targets for motion-impaired computer users. In: Proceedings of Conference on Human Factors in Computing Systems, ACM Press (2003) 487

[6] Myers, B.A., Wobbrock, J.O., Yang, S., Yeung, B., Nichols, J., Miller, R.: Using handhelds to help people with motor impairments. In: Proceedings of Conference on Assistive technologies, ACM Press (2002) 487

[7] Ashraf, S., Judson, A., Ricketts, I.W., Waller, A., Alm, N., Gordon, B., MacAulay, F., Brodie, J.K., Etchels, M., Warden, A., Shearer, A.J.: Capturing phrases for icutalk, a communication aid for intubated intensive care patients. In: Proceedings of Conference on Assistive Technologies, ACM Press (2002) 487

[8] Story, M.F.: Maximising usability: The principles of universal design. Assistive Technology Journal 10 (1998) 4-12 490

[9] Stephanidis, C., Salvendy, G., Akoumianakis, D., Bevan, N., Brewer, J., Emiliani, P.L., Galetsas, A., Haataja, S., Iakovidis, I., Jacko, J., Jenkins, P., Karshmer, A., Korn, P., Marcus, A., Murphy, H., Stary, C., Vanderheiden, G., Weber, G., Ziegler, J.: Towards an information society for all. International Journal of HumanComputer Interaction 10 (1998) 107-134 490 